\title{
Differential Effect of Light Emitting Diode Light on Electroencephalographic Oscillations in Young and Old Healthy Subjects
}

\author{
Jun-Sang Sunwoo ${ }^{1}$, Sanghun Lee ${ }^{2,3}$, Kwang Su Cha ${ }^{2}$, Tae-Joon Kim ${ }^{2,4}$, Jung-Ick Byun ${ }^{5}$, Ki-Young Jung 2,4,6 \\ ${ }^{1}$ Department of Neurology, Soonchunhyang University College of Medicine, Seoul, \\ ${ }^{2}$ Department of Neurology, Seoul National University Hospital, Seoul, \\ ${ }^{3}$ Department of Mathematical Sciences, Korea Advanced Institute of Science and Technology, Daejeon, \\ ${ }^{4}$ Program in Neuroscience, Seoul National University College of Medicine, Seoul, \\ ${ }^{5}$ Department of Neurology, Kyung Hee University Hospital at Gangdong, Seoul, \\ ${ }^{6}$ Neuroscience Research Institute, Seoul National University College of Medicine, Seoul, Korea
}

Received December 2, 2017

Revised December 21, 2017

Accepted December 22, 2017

Address for correspondence

Ki-Young Jung, MD, PhD

Department of Neurology,

Seoul National University

Hospital, Seoul National

University College of Medicine,

101 Daehak-ro, Jongno-gu,

Seoul 03080, Korea

Tel: $+82-2-2072-4988$

Fax: +82-2-2072-2474

E-mail: jungky@snu.ac.kr
Objectives: To investigate brain oscillatory characteristics according to brightness and color temperature of light emitting diode (LED) light in young and elderly subjects. Methods: We analyzed 22 young (age, $29.0 \pm 5.2$ years) and 23 elderly (age, $64.8 \pm 4.5$ years) healthy subjects. A LED light source was used with a combination of two color temperature (6,500 K vs. 3,000 K) and two brightness (700 lx vs. $300 \mathrm{~lx}$ ) conditions. Participants were exposed to each light condition in relaxed wakefulness. Then, we analyzed power spectral density and functional connectivity from eye-open electroencephalography. Results: A main effect of brightness on delta $(p=0.044)$ and theta $(p=0.038)$ power was significant in the elderly subjects. Bright light enhanced delta and theta power in the frontal region. By contrast, power spectral density of young subjects was affected by color temperature; high color temperature significantly increased beta-band power of the central region $(p=0.034)$. Regarding functional connectivity, a significant effect of color temperature was observed in delta $(p=0.006)$ and beta $(p=0.046)$ frequencies. High color temperature light enhanced beta connectivity of young subjects ( $p=0.007$ ), while not affecting that of elderly subjects $(p=0.979)$. Conclusions: The present study demonstrated that spectral power and functional connectivity as well as subjective feelings are affected by the brightness and color temperature of LED light. These results might help us to understand the neurophysiological effects of light and identify the optimal indoor lighting conditions for an individual's environment.

J Sleep Med 2017;14(2):61-69

Key Words: Power spectral density, Functional connectivity, Electroencephalography, Light emitting diode, Brightness, Color temperature.

\section{Introduction}

Light has profound effects on human health. ${ }^{1}$ Endogenous circadian rhythms affect physiological processes of major organ systems as cardiovascular, metabolic, and gastrointestinal functions as well as sleep-wake cycle. ${ }^{2}$ Light, known as a dominant zeitgeber, is essential for synchronization or entrainment of endogenous circadian system to the 24-hr lightdark cycle. ${ }^{3}$ Disruption of the circadian rhythm is associated with various diseases, such as insomnia, depression, cardioThis is an Open Access article distributed under the terms of the Creative Commons Attribution Non-Commercial License (http://creativecommons.org/licenses/by-nc/4.0) which permits unrestricted non-commercial use, distribution, and reproduction in any medium, provided the original work is properly cited. vascular diseases, and cancers. ${ }^{4-6}$ Moreover, light have an effect on mood, perception, and cognition. ${ }^{7.8}$ Brightness and color temperature are important lighting conditions affecting subjective perception and cognitive performance. Bright light exposure during the daytime reduces sleepiness and improves psychomotor vigilance task performance. ${ }^{7}$ Exposure to bright light was also reported to attenuate cognitive deterioration in elderly patients with dementia. ${ }^{9}$ On the other hand, color temperature is a measure of color appearance provided by a light source; low color temperature is red-enriched white with warm and stable mood, whereas high color temperature is red-enriched white with cool and active atmosphere ${ }^{10}$ High color temperature light was shown to increase levels of arousal and 
concentration as well as psychological well-being. ${ }^{11}$

Although there has been substantial research into the effects of lighting conditions on mood and behavior, previous investigations were mostly based on subjective measures such as self-reported questionnaires. Accordingly, there has been relatively little evidence objectively showing that brain activities are altered by different lighting conditions. Electroencephalography (EEG) is an electrophysiological method to record the brain activity and its change according to different lighting conditions. ${ }^{12}$ Because prior EEG studies were limited to investigations for young subjective, ${ }^{13-15}$ little is known about neurophysiological characteristics of the lighting effects in elderly subjects. Therefore, herein, we investigated brain oscillatory characteristics according to brightness and color temperature of light by analyzing power spectral density and functional connectivity derived from resting-state EEG recordings. Furthermore, we compared the lighting effects between young and elderly subjects to determine age-dependent responses to brightness and color temperature.

\section{Methods}

\section{Subjects}

We prospectively recruited healthy young $(n=30$, age range $20-39$ years) and old ( $n=30$, age range $60-76$ years) volunteers through advertisement from February to June 2016. Exclusion criteria were history of any neurological or psychiatric disorders, significant head trauma, and brain surgery. Those who took medication within the last two weeks, which can affect EEG recordings, such as antiepileptic drugs, sleeping pills, and antidepressants, were also excluded. All participants visited the Department of Neurology of the Seoul National University Hospital and were screened for the selection criteria. All subjects gave written informed consent before enrollment into the study. Among 60 subjects enrolled, 8 young and 7 old subjects were excluded from the analysis because of poor EEG data quality. Finally, data from 22 young (mean age, $29.0 \pm$ 5.2 years; men $50 \%$ ) and 23 elderly (mean age, $64.8 \pm 4.5$ years; men $82.6 \%$ ) subjects were analyzed in this study. This study was approved by the Institutional Review Board of the Seoul National University Hospital (H-1601-043-733) and was conducted in compliance with the Declaration of Helsinki and the Good Clinical Practice guidelines.

\section{Lighting condition}

We used four different light conditions as a combination of color temperature (6,500 K vs. 3,000 K) and brightness (700 lx vs. $300 \mathrm{~lx})$ : high-bright (6,500 K, $700 \mathrm{~lx})$; high-dark (6,500 K, $300 \mathrm{~lx})$; low-bright $(3,000 \mathrm{~K}, 700 \mathrm{~lx})$; and low-dark (3,000 K, $300 \mathrm{~lx}$ ) light. These light parameters were chosen within the range of indoor lighting conditions which are commonly used in the real world. The experimental space (width $2 \mathrm{~m}$, length $2.5 \mathrm{~m}$, height $2.7 \mathrm{~m}$ ) was shielded from ambient light. Light emitting diode (LED) light panels (Mimilighting, Incheon, Korea) were suspended from the ceiling. We used a controller to regulate color temperature and brightness of LED light per session. The lighting condition was checked at desk level $(0.7 \mathrm{~m})$.

For each light condition, subjects first took a rest in a lightless condition for $5 \mathrm{~min}$. Then, LED light of specific parameters was presented for $5 \mathrm{~min}$ when subjects opened and closed their eyes alternately every $1 \mathrm{~min}$ (Fig. 1A). EEG recordings during the last $4 \mathrm{~min}$ after the light was turned on were used for analysis of spectral power and functional connectivity. The order of the light conditions was counterbalanced across subjects (Fig. 1B). For 4 lighting condition with the repeated measures design, there are 24 orders of light exposure. We added 6 more orders to make 30 orders of lighting condition for 30 participants. Although it is not complete randomization, we used this counterbalance design to minimize the effect of the order of light condition exposed to the subjects. Subjective feelings on sleepiness, refreshment, comfort, and happiness were evaluated at the end of each light condition. The degree of sleepiness was evaluated using the Stanford sleepiness scale ranging from 1 to $7 .{ }^{16}$ Subjective refreshment, comfort, and happiness were measured by using 11-point Likert scales with 0 indicating "not at all" and 10 indicating "extremely".

\section{EEG recording and pre-processing}

EEG was recorded using a 64-channel recording system (Grass Technologies, Quincy, MA, USA) with 60 cap-based electrodes (Quick-Cap, Charlotte, NC, USA) that were located according to the international 10-20 system. Impedances were kept below $10 \mathrm{k} \Omega$ in all electrodes. EEG recordings were sampled at $400 \mathrm{~Hz}$ and referenced to an average reference. A bandpass filter was set between $0.5-70 \mathrm{~Hz}$. We thoroughly inspected EEG recordings and selected 30 artifactfree epochs of 2-s duration from eye-open resting-state EEG for each light condition.

Selected EEG data were pre-processed and further analyzed for power spectral density and functional connectivity using MATLAB (MathWorks, Natick, MA, USA). We applied 60-Hz notch filter and interpolated bad EEG channels. Then, we performed independent component analysis decomposition and removed components containing ocular movement, heartbeat, and muscle artifacts. The current source density transformation was employed to enhance EEG spatial resolution and minimize the volume conduction effect. In addition, EEG data were demeaned and detrended to remove nonstationarity of the time series mean. 

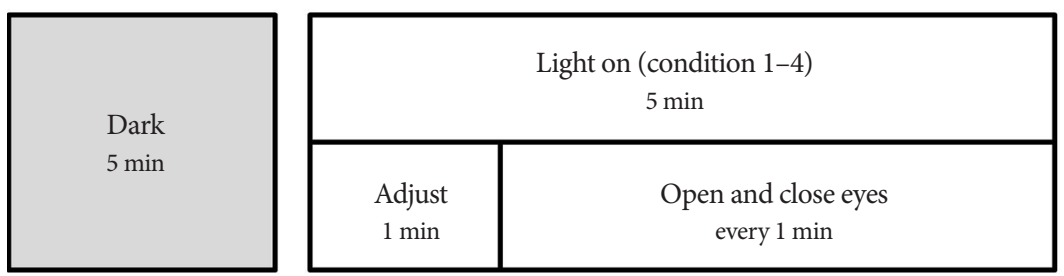

A

Order of lighting conditions

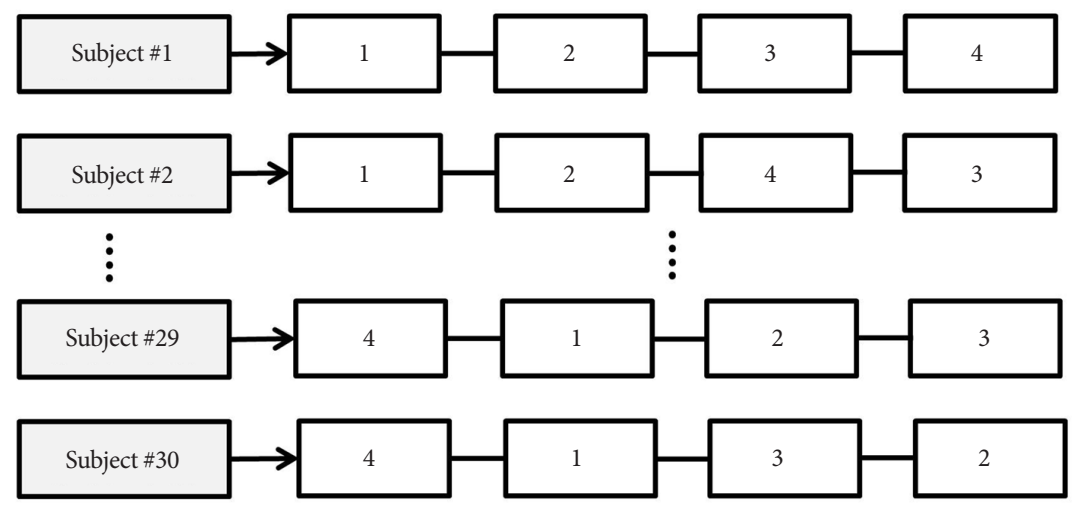

B

Figure 1. Experimental design. (A) Schematic illustration of exposure to each lighting condition. (B) Schematic illustration of a counterbalanced design for the order of lighting conditions. Each subject is exposed to all 4 lighting conditions in a different order.

\section{Power spectral analysis}

Spectral power analysis was performed using a fast Fourier transformation with the Welch's method (1-s hamming window, $50 \%$ overlap).$^{17}$ Frequencies were divided into delta $(0.5-4 \mathrm{~Hz})$, theta $(4-8 \mathrm{~Hz})$, alpha $(8-12 \mathrm{~Hz})$, and beta $(12-30$ $\mathrm{Hz}$ ) bands with a frequency resolution of $0.25 \mathrm{~Hz}$. Electrodes were grouped into three topographical regions as previously described $^{18}$ : the frontal (AF3, AFz, AF4, F5, F3, F1, Fz, F2, F4, and F6); central (FC5, FC3, FC1, FCz, FC2, FC4, FC6, C5, $\mathrm{C} 3, \mathrm{C} 1, \mathrm{Cz}, \mathrm{C} 2, \mathrm{C} 4, \mathrm{C} 6, \mathrm{CP} 5, \mathrm{CP} 3, \mathrm{CP} 1, \mathrm{CPz}, \mathrm{CP} 2, \mathrm{CP} 4$, and $\mathrm{CP6}$ ); and the parietal (P5, P3, P1, Pz, P2, P4, P6, PO3, POz, and $\mathrm{PO} 4$ ) regions. Power spectral density was expressed in $\mathrm{dB}$.

\section{Functional connectivity analysis}

To measure EEG functional connectivity, we computed the weighted phase lag index (wPLI). ${ }^{19}$ The wPLI is an improved index of phase synchronization compared to the phase lag index (PLI) in terms of increased power to detect changes in phase synchronization as well as reduced sensitivity to uncorrelated noise sources. ${ }^{20}$ For functional connectivity analysis, we only used EEG data recorded from 21 electrodes according to the international 10-20 system. Briefly, the Hilbert transformation was applied to calculate the instantaneous phase for each time point. Then, we measured functional connectivity by averaging wPLI values over all 210 electrode pairs. The average wPLI for each subject was estimated for four fre- quency bands: delta $(0.5-4 \mathrm{~Hz})$; theta $(4-8 \mathrm{~Hz})$; alpha $(8-12$ $\mathrm{Hz})$; and beta $(12-30 \mathrm{~Hz})$ bands.

\section{Statistical analysis}

For power spectral density, we conducted repeated-measures analysis of variance (ANOVA). Within-subject variables included brightness (two levels: bright and dark), color temperature (two levels: high and low), and brain regions (three levels: frontal, central and parietal), while a betweensubject variable was age groups (two levels: old and young). Thereafter, we conducted repeated-measures ANOVA per each age group. For functional connectivity and subjective evaluation data, within-subject variables included brightness (two levels: bright and dark) and color temperature (two levels: high and low), and between-subject variables were age groups (two levels: old and young). Both power spectral density and functional connectivity data were analyzed for each frequency band. When sphericity assumptions were violated, we applied the Greenhouse-Geisser correction to control for type 1 error rates. Multiple comparisons in post hoc analysis were corrected by the Bonferroni method. A two-tailed $p<0.05$ was considered statistically significant. Statistical analyses were performed using SPSS version 18.0 (SPSS Inc., Chicago, IL, USA). 


\section{Results}

\section{Power spectral density of overall subjects}

A significant interaction between brightness and age group was noted in theta power $\left(\mathrm{F}_{1,43}=4.704, p=0.036\right)$. In addition, there was a marginal interaction between brightness and group in delta frequency $\left(\mathrm{F}_{1,43}=3.596, p=0.065\right)$. However, for color temperature, no significant interaction effect with age group was observed in all frequencies. The results of repeatedmeasures ANOVA are summarized in Supplementary Table 1 (in the online-only Data Supplement).

\section{Power spectral density of the elderly subjects}

A main effect of brightness on delta-band power was significant in the old age group $\left(\mathrm{F}_{1,22}=4.545, p=0.044\right)$ (Table 1$)$; bright light increased delta-band power compared to dark light. The effect of bright light was particularly significant in the frontal region ( $p=0.013$ ) (Fig. $2 \mathrm{~A}$ ). However, the main effect of color temperature $\left(\mathrm{F}_{1,22}=0.639, p=0.433\right)$ and the interaction between brightness and color temperature $\left(\mathrm{F}_{1,22}=\right.$ $1.293, p=0.268$ ) were not significant.

Similarly, theta-band power were significantly higher in bright light than in dark light $\left(\mathrm{F}_{1,22}=4.882, p=0.038\right)$. Analysis per region revealed that bright light particularly affected the frontal region ( $p=0.031$ ) (Fig. 2B). However, there was no sig- nificant main effect of color temperature $\left(\mathrm{F}_{1,22}=0.166, p=0.687\right)$ or the interaction between brightness and color temperature $\left(\mathrm{F}_{1,22}=0.170, p=0.684\right)$.

By contrast, power spectral density of alpha, beta bands were not affected by brightness or color temperature. In addition, there was no significant interaction between brightness and color temperature at these frequencies. Taken together, power spectral density of elderly subjects was influenced by brightness rather than color temperature. Bright light enhanced the delta- and theta-band power particularly in the frontal region.

\section{Power spectral density of the young subjects}

We observed a marginally significant effect of color temperature on beta power $\left(\mathrm{F}_{1,21}=3.839, p=0.063\right)$ (Table 2). Especially, high color temperature significantly increased betaband power of the central region compared with low color temperature ( $p=0.034$ ) (Fig. 3A). However, a main effect of brightness was not significant $\left(\mathrm{F}_{1,21}=0.030, p=0.863\right)$. Moreover, there was a significant interaction between brightness and color temperature $\left(\mathrm{F}_{1,21}=6.484, p=0.019\right)$. As shown in Fig. $3 \mathrm{~B}$, color temperature significantly affected the beta power under dark light $(p=0.012)$ but not under bright light $(p=0.574)$. Furthermore, post-hoc analyses revealed that high color temperature increased the beta-band power of frontal $(p=0.012)$

Table 1. Repeated-measures analysis of variance for power spectral density in elderly subjects

\begin{tabular}{|c|c|c|c|c|c|c|c|c|}
\hline \multirow{2}{*}{ Factors } & \multicolumn{2}{|c|}{ Delta } & \multicolumn{2}{|c|}{ Theta } & \multicolumn{2}{|c|}{ Alpha } & \multicolumn{2}{|c|}{ Beta } \\
\hline & $\mathrm{F}$ & $p$ & $\mathrm{~F}$ & $p$ & $\mathrm{~F}$ & $p$ & $\mathrm{~F}$ & $p$ \\
\hline Color temperature & 0.639 & 0.433 & 0.166 & 0.687 & 0.168 & 0.686 & $<0.001$ & 0.984 \\
\hline Brightness & 4.545 & $0.044^{*}$ & 4.882 & $0.038^{*}$ & 0.503 & 0.486 & 0.379 & 0.545 \\
\hline Region & 2.117 & 0.151 & 6.743 & $0.003^{* *}$ & 33.176 & $<0.001^{* *}$ & 0.841 & 0.417 \\
\hline Color temperature $\times$ brightness & 1.293 & 0.268 & 0.170 & 0.684 & 0.798 & 0.381 & 0.114 & 0.739 \\
\hline Region $\times$ color temperature $\times$ brightness & 1.105 & 0.326 & 0.350 & 0.707 & 0.804 & 0.420 & 0.057 & 0.906 \\
\hline
\end{tabular}

Degree of freedom $=1,22 .{ }^{*} p<0.05,{ }^{* *} p<0.01$
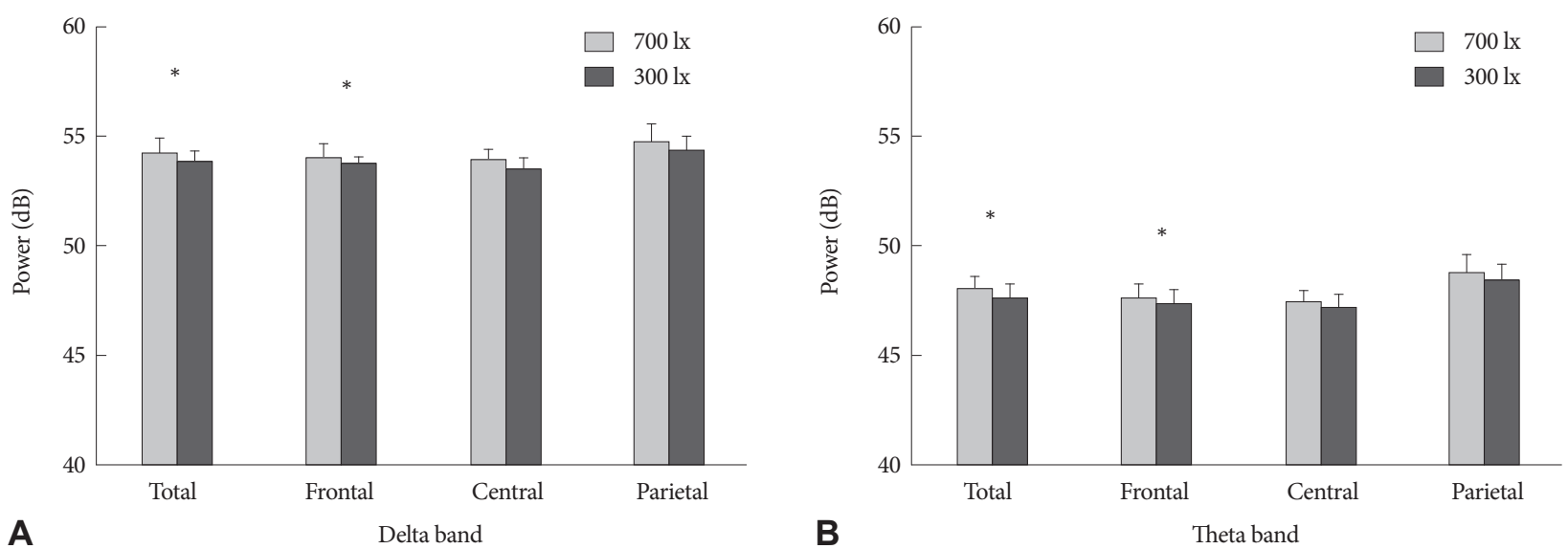

Figure 2. Power spectral density of the elderly subjects according to brightness of light. (A) Delta-band (0.5-4 Hz) power. (B) Theta-band (4-8 Hz) power. Error bars indicate the standard errors of the mean. $n=23 .{ }^{*} p<0.05$. 
and central $(p=0.016)$ regions compared with low color temperature under dark light.

At delta, theta, and alpha frequencies, however, there was no significant effect of different light conditions on power spectral density. In summary, power spectral density of young subjects was mainly affected by color temperature; high color temperature increased beta-band power in the central regions. Further, these effects of color temperature were partic- ularly significant under the dark light condition.

\section{Functional connectivity analysis}

A significant main effect of color temperature on functional connectivity was observed in delta $\left(\mathrm{F}_{1,43}=8.428, p=0.006\right)$ and beta $\left(\mathrm{F}_{1,43}=4.207, p=0.046\right)$ frequencies; high color temperature light increased EEG functional connectivity compared to low color temperature light (Fig. 4A). Furthermore, we

Table 2. Repeated-measures analysis of variance for power spectral density in young subjects

\begin{tabular}{|c|c|c|c|c|c|c|c|c|}
\hline \multirow{2}{*}{ Factors } & \multicolumn{2}{|c|}{ Delta } & \multicolumn{2}{|c|}{ Theta } & \multicolumn{2}{|c|}{ Alpha } & \multicolumn{2}{|c|}{ Beta } \\
\hline & $\mathrm{F}$ & $p$ & $\mathrm{~F}$ & $p$ & $\mathrm{~F}$ & $p$ & $\mathrm{~F}$ & $p$ \\
\hline Color temperature & 1.219 & 0.282 & 3.238 & 0.086 & 1.026 & 0.323 & 3.839 & 0.063 \\
\hline Brightness & 0.465 & 0.503 & 1.168 & 0.292 & 1.780 & 0.196 & 0.030 & 0.863 \\
\hline Region & 25.447 & $<0.001^{* *}$ & 26.333 & $<0.001^{* *}$ & 14.033 & $<0.001^{* *}$ & 26.406 & $<0.001^{* *}$ \\
\hline Color temperature $\times$ brightness & 0.015 & 0.904 & 0.158 & 0.695 & 1.652 & 0.213 & 6.484 & $0.019^{*}$ \\
\hline Region $\times$ color temperature $\times$ brightness & 0.102 & 0.903 & 1.322 & 0.274 & 0.210 & 0.680 & 4.816 & $0.023^{*}$ \\
\hline
\end{tabular}

Degree of freedom $=1,21 .{ }^{*} p<0.05,{ }^{* *} p<0.01$

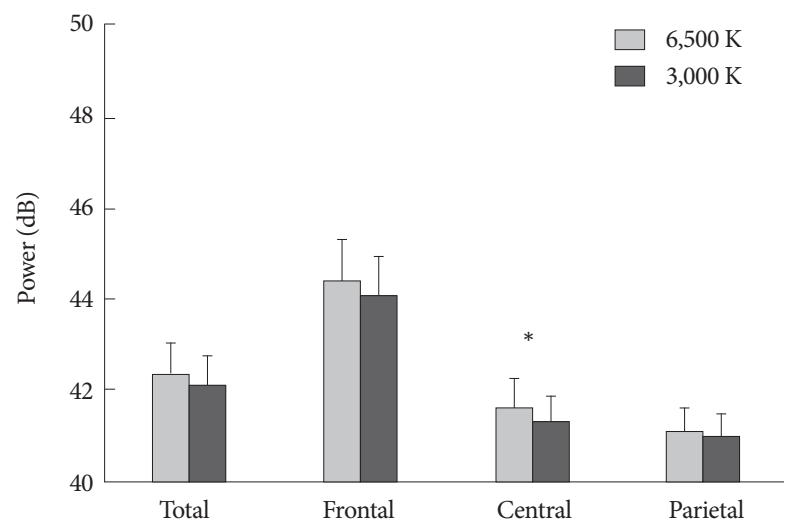

A

Figure 3. Power spectral density of the young subjects according to color temperature of light. (A) Beta-band (12-30 Hz) power per brain region. (B) Interaction between color temperature and brightness on beta power. Error bars indicate the standard errors of the mean. $n=22$. ${ }^{*} p<0.05$.

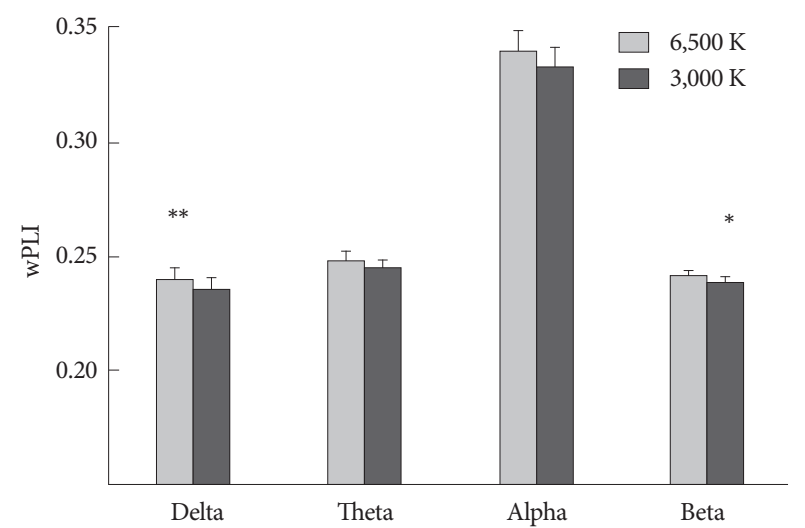

A

Frequency band

Figure 4. Effect of color temperature on functional connectivity. (A) Comparisons of wPLI for each frequency band. $n=45$. (B) Beta-band functional connectivity for each age group. Error bars indicate the standard errors of the mean. Old group, $n=23$; Young group, $n=22$. ${ }^{*} p<0.05,{ }^{* *} p<0.01$. wPLI: weighted phase lag index.

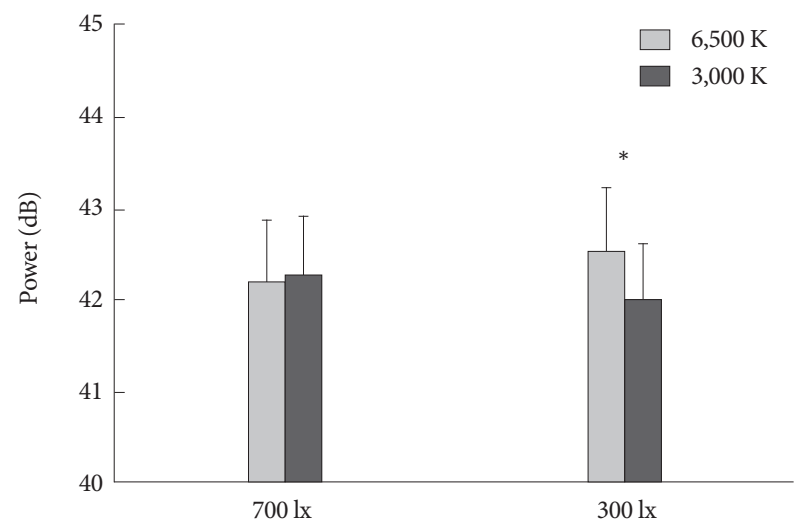

Beta band

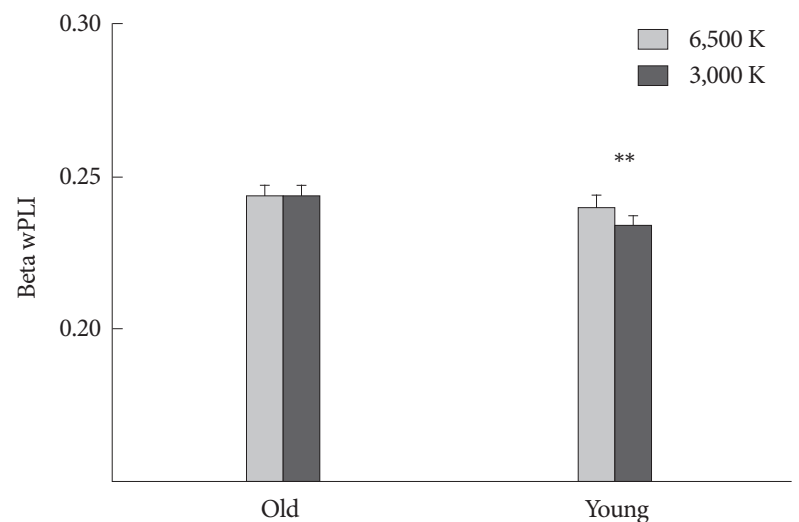


observed an interaction between color temperature and group at beta frequency $\left(\mathrm{F}_{1,43}=4.508, p=0.050\right)$. Post-hoc comparisons revealed that high color temperature enhanced the betaband connectivity of young subjects $(p=0.007$ ), while not affecting that of elderly subjects $(p=0.979)$ (Fig. 4B). As opposed to color temperature, brightness did not affect functional connectivity and there was no significant interaction between brightness and group in all frequency bands (Table 3 ).

\section{Subjective evaluations}

There was a significant effect of color temperature on comfort $\left(\mathrm{F}_{1,43}=7.302, p=0.010\right)$, happiness $\left(\mathrm{F}_{1,43}=4.361, p=0.043\right)$, and refreshment $\left(\mathrm{F}_{1,43}=4.166, p=0.047\right)$. Subjects felt more refreshed in high temperature conditions while they reported

Table 3. Summary of repeated-measures analysis of variance for functional connectivity

\begin{tabular}{|c|c|c|c|c|c|c|c|c|}
\hline \multirow{2}{*}{ Factors } & \multicolumn{2}{|c|}{ Delta } & \multicolumn{2}{|c|}{ Theta } & \multicolumn{2}{|c|}{ Alpha } & \multicolumn{2}{|c|}{ Beta } \\
\hline & $\mathrm{F}$ & $p$ & $\mathrm{~F}$ & $p$ & $\mathrm{~F}$ & $p$ & $\mathrm{~F}$ & $p$ \\
\hline Color temperature & 8.428 & $0.006^{* *}$ & 2.842 & 0.099 & 3.242 & 0.079 & 4.207 & $0.046^{*}$ \\
\hline Color temperature $\times$ group & 1.175 & 0.284 & 1.273 & 0.265 & 0.314 & 0.578 & 4.058 & 0.050 \\
\hline Brightness & 2.031 & 0.161 & 0.023 & 0.881 & 0.508 & 0.480 & 0.272 & 0.605 \\
\hline Brightness $\times$ group & 0.137 & 0.714 & 0.018 & 0.893 & 2.013 & 0.163 & 0.245 & 0.623 \\
\hline Color temperature $\times$ brightness & 0.016 & 0.900 & 1.808 & 0.186 & 0.177 & 0.676 & 2.916 & 0.096 \\
\hline Color temperature $\times$ brightness $\times$ group & 0.015 & 0.902 & 0.096 & 0.759 & 0.515 & 0.477 & 0.407 & 0.527 \\
\hline Group & 2.258 & 0.140 & 0.281 & 0.599 & 0.225 & 0.637 & 2.171 & 0.148 \\
\hline
\end{tabular}

Degree of freedom $=1,43 .{ }^{*} p<0.05,{ }^{* *} p<0.01$
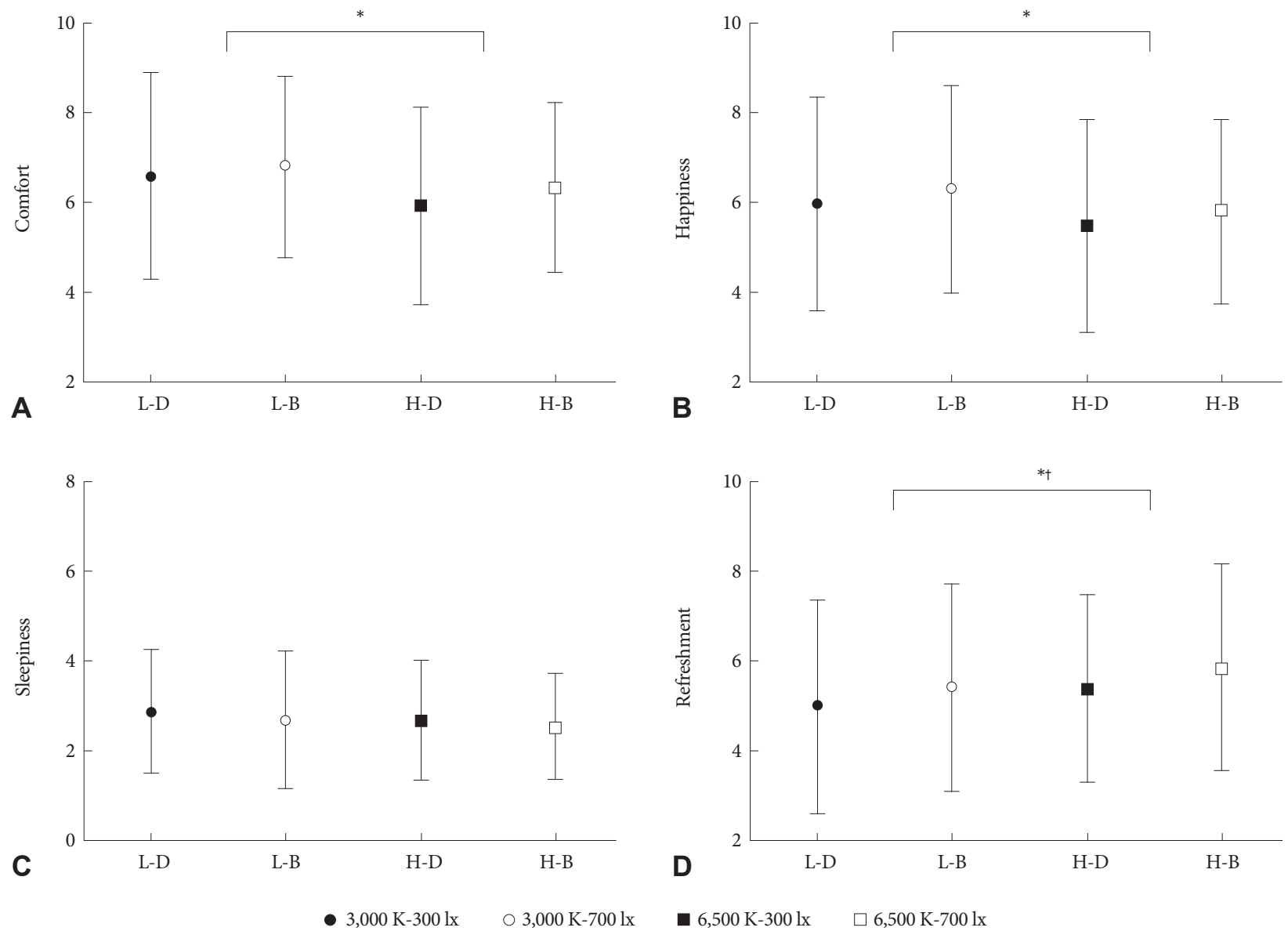

Figure 5. Subjective feelings according to different light conditions. (A) Comfort. (B) Happiness. (C) Sleepiness. (D) Refreshment. Error bars indicate standard deviations. $n=45 .{ }^{*} p<0.05$ for comparisons between high $(6,500 \mathrm{~K})$ and low $(3,000 \mathrm{~K})$ color temperature light; ${ }^{\dagger} p<0.05$ for comparisons between bright $(700 \mathrm{~lx})$ and dark (300 Ix) light. L-D: low-dark (3,000 K-300 Ix), L-B: low-bright (3,000 K-700 Ix), H-D: high-dark (6,500 K-300 Ix), H-B: high-bright (6,500 K-700 Ix). 
more comfort and happiness in low temperature conditions (Fig. 5). A significant interaction between color temperature and group was not observed for all subjective feelings. In addition, we found a significant main effect of brightness $\left(\mathrm{F}_{1,43}=\right.$ 9.929, $p=0.003)$ and interaction effect between brightness and group $\left(\mathrm{F}_{1,43}=5.729, p=0.021\right)$ on refreshment. Bright light provided more refreshing atmosphere than dark light. However, this effect was significant in young subjects $(p<0.001)$ but not in elderly subjects $(p=0.591)$. For subjective measures of sleepiness, there was no significant effect of color temperature or brightness. The results of repeated-measures ANOVA for subjective feelings are summarized in Supplementary Table 2 (in the online-only Data Supplement).

\section{Discussion}

The present study demonstrated that brain oscillations were influenced by brightness and color temperature of the LED light. Subjective feelings were also affected by the different lighting conditions, which was in agreement with previous studies. ${ }^{13,15}$ The consistent findings suggests that the lighting conditions and exposure protocols were appropriate in our experiments. Regarding power spectral density, the elderly subjects was mainly affected by brightness, whereas young subjects was influenced by color temperature of the LED light. In terms of the oscillation frequency, we found differential responses between the young and old age groups; elderly subjects showed changes in delta and theta power, whereas young subjects were characterized by changes in beta power. Furthermore, we found that resting-state functional connectivity was affected by color temperature but not by brightness. Exposure to high color temperature light increased functional connectivity in delta and beta frequencies. To the best of our knowledge, this is the first study to determine the lighting effect on functional connections in the brain. These results might help us to understand the neurophysiological effects of the light and to develop tailored lighting conditions for human health and disease.

Brain oscillatory activity changes with age, which is characterized by general slowing of background EEG activity together with decrement in amplitude and power of alpha rhythms. ${ }^{21,22}$ Furthermore, accumulating evidence have suggested that healthy aging is associated with a gradual decrease in delta and theta power. ${ }^{23-25}$ Of particular interest in our study is that bright light increased the frontal delta and theta power for elderly subjects, while not affecting spectral power of young subjects. Slow wave power of resting-state EEG was reported to correlate positively with cognitive performance such as verbal memory, attention, and executive function exclusively in healthy elderly adults. ${ }^{25,26}$ These findings indicate that higher slow-frequency power is a neurophysiological marker of better cognitive function in healthy elderly subjects. Taken together with our observation, it is presumed that exposure to bright light in the elderly might facilitate brain activities related with cognitive processing. It is also supported by our result that enhancement of slow-frequency power under the bright light occurred in the frontal region. Previous studies have shown that frontal midline theta oscillations recorded in scalp EEG were generated by the anterior cingulate cortex, medial prefrontal cortex, and hippocampus. ${ }^{27-29}$ Furthermore, frontal theta oscillations are functionally implicated in cognitive operations, including working memory and episodic memory processes. ${ }^{30,31}$ These findings further support the hypothesis that the bright light in elderly subjects might contribute to enhancing brain activities associated with neurocognitive functions. However, an event-related potential study reported that the bright background light recued prestimulus alpha power and interrupted sustained attention performance..$^{32}$ Moreover, the bright illumination decreased the prestimulus theta power in the frontal region during working memory tasks while not affecting cognitive performances. ${ }^{33}$ These results are in contrast to our results. However, previous studies investigated brain oscillations during cognitive tasks in young participants, so that they cannot be directly compared to our findings derived from the resting-state EEG of elderly subjects. Further study is needed to understand the neurophysiological mechanisms and functional significance of age-dependent EEG responses to the bright illumination.

Another significant finding in this study was that color temperature conditions modulated resting-state functional connectivity. High color temperature light increased overall connectivity strength particularly in delta and beta frequencies. Physiological effects of color temperature on brain activity have been evaluated by recording EEG under different light conditions. ${ }^{13,143,34,35}$ The previous studies consistently concluded that high color temperature light enhanced arousal levels and brain activity compared with low color temperature light. Furthermore, results from subjective evaluation were in close agreement with EEG findings; subjective sleepiness was higher under low color temperature light while the level of concentration was higher under high color temperature condition. ${ }^{13,14,34,35}$ Deguchi and Sato postulated that the brain activating effect of higher color temperature light might be mediated by the ascending reticular activation system, ${ }^{35}$ but physiological mechanisms remains unclear. From the viewpoint of functional connectivity, the present study provides a new insight into the effects of high color temperature light on brain activities. Functional networks for integrating separate brain regions are implicated in efficient cognitive abilities including attention. ${ }^{36-38}$ Moreover, functional connectivity 
of resting-state networks was reported to significantly correlate with attention performance during cognitive tasks. ${ }^{39,40}$ Accordingly, these findings support the notion that enhanced functional connectivity by the high color temperature light might contribute to higher arousal and attention levels. In this regard, the high color temperature lighting could be helpful in environments requiring higher concentration and attention, such as office, laboratory, and school. Further studies using cognitively demanding tasks will be required to validate functional significance of hypersynchronization effect by high color temperature light.

There are several limitations in this study. Brain oscillatory characteristics shown in this study represented short-term responses to the light conditions with exposure time of five min. Therefore, long-term effects of the light conditions cannot be concluded from this study. Another limitation is that subjective feelings except for sleepiness were not measured by validated scales. Moreover, the interaction effects between light conditions and age groups appeared to be modest with marginal significance levels. The subtle differences between the different age groups might be attributed to relatively short exposure to the light. Another possible explanation is that the resting state was not the optimal condition to detect brain oscillatory responses to the different light. In addition, we detected the changes in resting-state brain oscillations without neuropsychological tests, so that we could not determine that the altered EEG activities have functional implications particularly in neurocognitive performances. Further research such as cognitive event-related potentials under different light conditions will be required to address these concerns.

\section{Supplementary Materials}

The online-only Data Supplement is available with this article at https:// doi.org/10.13078/jsm.17009.

\section{Acknowledgments}

The authors would like to thank Byeong Uk Lee for his contribution to EEG recording and Min Hee Jeong for her contribution to EEG analysis. This work was supported by a research grant from Mimi Lighting Inc. (grant No. 06-2016-0560).

\section{REFERENCES}

1. Chepesiuk R. Missing the dark: health effects of light pollution. Environ Health Perspect 2009;117:A20-A27.

2. Morris CJ, Yang JN, Scheer FA. The impact of the circadian timing system on cardiovascular and metabolic function. Prog Brain Res 2012; 199:337-358.

3. Dijk DJ, Czeisler CA. Paradoxical timing of the circadian rhythm of sleep propensity serves to consolidate sleep and wakefulness in humans. Neurosci Lett 1994;166:63-68.

4. Pandi-Perumal SR, Moscovitch A, Srinivasan V, Spence DW, Cardinali DP, Brown GM. Bidirectional communication between sleep and circadian rhythms and its implications for depression: lessons from agomelatine. Prog Neurobiol 2009;88:264-271.
5. Chen L, Yang G. Recent advances in circadian rhythms in cardiovascular system. Front Pharmacol 2015;6:71.

6. Leonardi GC, Rapisarda V, Marconi A, et al. Correlation of the risk of breast cancer and disruption of the circadian rhythm (Review). Oncol Rep 2012;28:418-428.

7. Phipps-Nelson J, Redman JR, Dijk DJ, Rajaratnam SM. Daytime exposure to bright light, as compared to dim light, decreases sleepiness and improves psychomotor vigilance performance. Sleep 2003;26:695-700.

8. Hawes BK, Brunyé TT, Mahoney CR, Sullivan JM, Aall CD. Effects of four workplace lighting technologies on perception, cognition and affective state. Int J Ind Ergon 2012;42:122-128.

9. Riemersma-van der Lek RF, Swaab DF, Twisk J, Hol EM, Hoogendijk WJ, Van Someren EJ. Effect of bright light and melatonin on cognitive and noncognitive function in elderly residents of group care facilities: a randomized controlled trial. JAMA 2008;299:2642-2655.

10. Küller R, Wetterberg L. Melatonin, cortisol, EEG, ECG and subjective comfort in healthy humans: impact of two fluorescent lamp types at two light intensities. Lighting Research \& Technology 1993;25:71-80.

11. Mills PR, Tomkins SC, Schlangen LJ. The effect of high correlated colour temperature office lighting on employee wellbeing and work performance. J Circadian Rhythms 2007;5:2.

12. Lee GT, Lee C, Kim D, Lee H, Woo S, Jung KY. Effects of light-emitting diode light on human electroencephalogram in comparison with fluorescent light. J Korean Sleep Res Soc 2012;9:28-33.

13. Shi L, Katsuura T, Shimomura Y, Iwanaga K. Effects of different light source color temperatures during physical exercise on human EEG and subjective evaluation. J Hum Environ Syst 2009;12:27-34.

14. Katsuura T, Jin X, Baba Y, Shimomura Y, Iwanaga K. Effects of color temperature of illumination on physiological functions. J Physiol Anthropol Appl Human Sci 2005;24:321-325.

15. Park JY, Ha RY, Ryu V, Kim E, Jung YC. Effects of color temperature and brightness on electroencephalogram alpha activity in a polychromatic light-emitting diode. Clin Psychopharmacol Neurosci 2013;11: 126-131.

16. Hoddes E, Zarcone V, Smythe H, Phillips R, Dement WC. Quantification of sleepiness: a new approach. Psychophysiology 1973;10:431-436.

17. Welch P. The use of fast Fourier transform for the estimation of power spectra: a method based on time averaging over short, modified periodograms. IEEE Transactions on Audio and Electroacoustics 1967;15: 70-73

18. Moazami-Goudarzi M, Sarnthein J, Michels L, Moukhtieva R, Jeanmonod D. Enhanced frontal low and high frequency power and synchronization in the resting EEG of parkinsonian patients. Neuroimage 2008;41:985-997.

19. Vinck M, Oostenveld R, van Wingerden M, Battaglia F, Pennartz CM. An improved index of phase-synchronization for electrophysiological data in the presence of volume-conduction, noise and sample-size bias. Neuroimage 2011;55:1548-1565.

20. Sunwoo JS, Lee S, Kim JH, et al. Altered functional connectivity in idiopathic rapid eye movement sleep behavior disorder: a resting-state EEG study. Sleep 2017;40:zsx058. https://doi.org/10.1093/sleep/zsx058.

21. Rossini PM, Rossi S, Babiloni C, Polich J. Clinical neurophysiology of aging brain: from normal aging to neurodegeneration. Prog Neurobiol 2007;83:375-400.

22. Babiloni C, Binetti G, Cassarino A, et al. Sources of cortical rhythms in adults during physiological aging: a multicentric EEG study. Hum Brain Mapp 2006;27:162-172.

23. Cummins TD, Finnigan S. Theta power is reduced in healthy cognitive aging. Int J Psychophysiol 2007;66:10-17.

24. Leirer VM, Wienbruch C, Kolassa S, Schlee W, Elbert T, Kolassa IT. Changes in cortical slow wave activity in healthy aging. Brain Imaging Behav 2011;5:222-228.

25. Vlahou EL, Thurm F, Kolassa IT, Schlee W. Resting-state slow wave power, healthy aging and cognitive performance. Sci Rep 2014;4:5101.

26. Finnigan S, Robertson IH. Resting EEG theta power correlates with 
cognitive performance in healthy older adults. Psychophysiology 2011;48:1083-1087.

27. Onton J, Delorme A, Makeig S. Frontal midline EEG dynamics during working memory. Neuroimage 2005;27:341-356.

28. Asada H, Fukuda Y, Tsunoda S, Yamaguchi M, Tonoike M. Frontal midline theta rhythms reflect alternative activation of prefrontal cortex and anterior cingulate cortex in humans. Neurosci Lett 1999;274:29-32.

29. Mitchell DJ, McNaughton N, Flanagan D, Kirk IJ. Frontal-midline theta from the perspective of hippocampal "theta". Prog Neurobiol 2008; 86:156-185.

30. Hsieh LT, Ranganath C. Frontal midline theta oscillations during working memory maintenance and episodic encoding and retrieval. Neuroimage 2014;85 Pt 2:721-729.

31. Zakrzewska MZ, Brzezicka A. Working memory capacity as a moderator of load-related frontal midline theta variability in Sternberg task. Front Hum Neurosci 2014;8:399.

32. Min BK, Jung YC, Kim E, Park JY. Bright illumination reduces parietal EEG alpha activity during a sustained attention task. Brain Res 2013; 1538:83-92.

33. Park JY, Min BK, Jung YC, Pak H, Jeong YH, Kim E. Illumination influ- ences working memory: an EEG study. Neuroscience 2013;247:386-394.

34. Noguchi H, Sakaguchi T. Effect of illuminance and color temperature on lowering of physiological activity. Appl Human Sci 1999;18:117123.

35. Deguchi T, Sato M. The effect of color temperature of lighting sources on mental activity level. Ann Physiol Anthropol 1992;11:37-43.

36. Corbetta M, Shulman GL. Control of goal-directed and stimulus-driven attention in the brain. Nat Rev Neurosci 2002;3:201-215.

37. Parks EL, Madden DJ. Brain connectivity and visual attention. Brain Connect 2013;3:317-338.

38. Greicius MD, Krasnow B, Reiss AL, Menon V. Functional connectivity in the resting brain: a network analysis of the default mode hypothesis. Proc Natl Acad Sci U S A 2003;100:253-258.

39. Rosenberg MD, Finn ES, Scheinost D, et al. A neuromarker of sustained attention from whole-brain functional connectivity. Nat Neurosci 2016;19:165-171.

40. Baldassarre A, Lewis CM, Committeri G, Snyder AZ, Romani GL, Corbetta M. Individual variability in functional connectivity predicts performance of a perceptual task. Proc Natl Acad Sci U S A 2012;109: 3516-3521. 